\title{
On hybrid split problem and its nonlinear algorithms
}

\section{Zhenhua $\mathrm{He}^{1}$ and Wei-Shih Du ${ }^{2 *}$}

${ }^{*}$ Correspondence:

wsdu@nknucc.nknu.edu.tw

2Department of Mathematics, National Kaohsiung Normal

University, Kaohsiung, 824, Taiwan

Full list of author information is

available at the end of the article

\begin{abstract}
In this paper, we study a hybrid split problem (HSP for short) for equilibrium problems and fixed point problems of nonlinear operators. Some strong and weak convergence theorems are established.

MSC: 47J25; 47H09;65K10

Keywords: fixed point problem; equilibrium problem; hybrid split problem; iterative algorithm; strong (weak) convergence theorem
\end{abstract}

\section{Introduction}

Throughout this paper, we assume that $H$ is a real Hilbert space with zero vector $\theta$, whose inner product and norm are denoted by $\langle\cdot, \cdot\rangle$ and $\|\cdot\|$, respectively. Let $C$ be a nonempty subset of $H$ and $T: C \rightarrow H$ be a mapping. Denote by $\mathcal{F}(T)$ the set of fixed points of $T$. The symbols $\mathbb{N}$ and $\mathbb{R}$ are used to denote the sets of positive integers and real numbers, respectively.

Let $H$ be a Hilbert space and $C$ be a closed convex subset of $H$. Let $f: C \times C \rightarrow \mathbb{R}$ be a bi-function. The classical equilibrium problem (EP for short) is defined as follows.

$$
\text { Find } p \in C \text { such that } f(p, y) \geq 0, \quad \forall y \in C \text {. }
$$

The symbol $E P(f)$ is used to denote the set of all solutions of the problem (EP), that is,

$$
E P(f)=\{u \in K: f(u, v) \geq 0, \forall v \in K\}
$$

It is known that the problem (EP) contains optimization problems, complementary problems, variational inequalities problems, saddle point problems, fixed point problems, bilevel problems, semi-infinite problems and others as special cases and have many applications in physics and economics problems; for detail, one can refer to [1-3] and references therein.

In last ten years or so, the problem (EP) has been generalized and improved to find a common element of the set of fixed points of a nonlinear operator and the set of solutions of the problem (EP). More precisely, many authors have studied the following problem (FTEP) (see, for instance, [4-9]):

$$
\text { Find } p \in C \text { such that } T p=p \text { and } f(p, y) \geq 0, \quad \forall y \in C,
$$


where $C$ is a closed convex subset of a Hilbert space $H, f: C \times C \rightarrow \mathbb{R}$ is a bi-function and $T: C \rightarrow C$ is a nonlinear operator.

In this paper, motivated by the problems (EP) and (FTEP), we study the following mathematical model about a hybrid split problem for equilibrium problems and fixed point problems of nonlinear operators (HSP for short).

Let $E_{1}$ and $E_{2}$ be two real Banach spaces. Let $C$ be a closed convex subset of $E_{1}$ and $K$ be a closed convex subset of $E_{2}$. Let $f: C \times C \rightarrow \mathbb{R}$ and $g: K \times K \rightarrow \mathbb{R}$ be two bifunctions, $A: E_{1} \rightarrow E_{2}$ be a bounded linear operator. Let $T: C \rightarrow C$ and $S: K \rightarrow K$ be two nonlinear operators with $\mathcal{F}(T) \neq \emptyset$ and $\mathcal{F}(S) \neq \emptyset$. The mathematical model about a hybrid split problem for equilibrium problems and fixed point problems of nonlinear operators (HSP for short) is defined as follows:

$$
\begin{aligned}
& \text { Find } p \in C \text { such that } T p=p, f(p, y) \geq 0, \quad \forall y \in C, \quad \text { and } \\
& u:=A p \text { satisfying } S u=u \in K, g(u, v) \geq 0, \quad \forall v \in K .
\end{aligned}
$$

In fact, (HSP) contains several important problems as special cases. We give some examples to explain about it.

Example A If $T$ is an identity operator on $C$, then (HSP) will reduce to the following problem $\left(\mathrm{P}_{1}\right)$ :

$\left(\mathrm{P}_{1}\right)$ Find $p \in C$ such that $f(p, y) \geq 0, \forall y \in C$, and $u:=A p$ satisfying $S u=u \in K, g(u, v) \geq 0$, $\forall v \in K$.

Example B If $S$ is an identity operator on $K$, then (HSP) will reduce to the following problem $\left(\mathrm{P}_{2}\right)$ :

$\left(\mathrm{P}_{2}\right)$ Find $p \in C$ such that $T p=p, f(p, y) \geq 0, \forall y \in C$, and $u:=A p \in K$ satisfying $g(u, v) \geq 0$, $\forall v \in K$.

Example C If $T, S$ are all identity operators, then (HSP) will reduce to the following split equilibrium problem $\left(\mathrm{P}_{3}\right)$ which has been considered in [10]:

$\left(\mathrm{P}_{3}\right)$ Find $p \in C$ such that $f(p, y) \geq 0, \forall y \in C$, and $u:=A p \in K$ satisfying $g(u, v) \geq 0, \forall v \in K$.

Example D If $S$ is an identity operator and $f(x, y) \equiv 0$ for all $(x, y) \in C \times C$, then (HSP) will reduce to the following problem $\left(\mathrm{P}_{4}\right)$ which has been studied in [11]:

$\left(\mathrm{P}_{4}\right)$ Find $p \in C$ such that $T p=p$ and $u:=A p \in K$ satisfying $g(u, v) \geq 0, \forall v \in K$.

In this paper, we introduce some new iterative algorithms for (HSP) and some strong and weak convergence theorems for (HSP) will be established.

\section{Preliminaries}

In what follows, the symbols $\rightarrow$ and $\rightarrow$ will symbolize weak convergence and strong convergence as usual, respectively. A Banach space $(X,\|\cdot\|)$ is said to satisfy Opial's condition if for each sequence $\left\{x_{n}\right\}$ in $X$ which converges weakly to a point $x \in X$, we have

$$
\liminf _{n \rightarrow \infty}\left\|x_{n}-x\right\|<\liminf _{n \rightarrow \infty}\left\|x_{n}-y\right\|, \quad \forall y \in X, y \neq x
$$


It is well known that any Hilbert space satisfies Opial's condition. Let $K$ be a nonempty subset of real Hilbert spaces $H$. Recall that a mapping $T: K \rightarrow K$ is said to be nonexpansive if $\|T x-T y\| \leq\|x-y\|$ for all $x, y \in K$.

Let $H_{1}$ and $H_{2}$ be two Hilbert spaces. Let $A: H_{1} \rightarrow H_{2}$ and $B: H_{2} \rightarrow H_{1}$ be two bounded linear operators. $B$ is called the adjoint operator (or adjoint) of $A$ if for all $z \in H_{1}, w \in H_{2}$, $B$ satisfies $\langle A z, w\rangle=\langle z, B w\rangle$. It is known that the adjoint operator of a bounded linear operator on a Hilbert space always exists and is bounded linear and unique. Moreover, it is not hard to show that if $B$ is an adjoint operator of $A$, then $\|A\|=\|B\|$.

Example 2.1 ([10]) Let $H_{2}=\mathbb{R}$ with the standard norm $|\cdot|$ and $H_{1}=\mathbb{R}^{2}$ with the norm $\|\alpha\|=\left(a_{1}^{2}+a_{2}^{2}\right)^{\frac{1}{2}}$ for some $\alpha=\left(a_{1}, a_{2}\right) \in \mathbb{R}^{2} .\langle x, y\rangle=x y$ denotes the inner product of $H_{2}$ for some $x, y \in H_{2}$ and $\langle\alpha, \beta\rangle=\sum_{i=1}^{2} a_{i} b_{i}$ denotes the inner product of $H_{1}$ for some $\alpha=$ $\left(a_{1}, a_{2}\right), \beta=\left(b_{1}, b_{2}\right) \in H_{1}$. Let $A \alpha=a_{2}-a_{1}$ for $\alpha=\left(a_{1}, a_{2}\right) \in H_{1}$ and $B x=(-x, x)$ for $x \in H_{2}$, then $B$ is an adjoint operator of $A$.

Example 2.2 ([10]) Let $H_{1}=\mathbb{R}^{2}$ with the norm $\|\alpha\|=\left(a_{1}^{2}+a_{2}^{2}\right)^{\frac{1}{2}}$ for some $\alpha=\left(a_{1}, a_{2}\right) \in \mathbb{R}^{2}$ and $H_{2}=\mathbb{R}^{3}$ with the norm $\|\gamma\|=\left(c_{1}^{2}+c_{2}^{2}+c_{3}^{2}\right)^{\frac{1}{2}}$ for some $\gamma=\left(c_{1}, c_{2}, c_{3}\right) \in \mathbb{R}^{3}$. Let $\langle\alpha, \beta\rangle=$ $\sum_{i=1}^{2} a_{i} b_{i}$ and $\langle\gamma, \eta\rangle=\sum_{i=1}^{3} c_{i} d_{i}$ denote the inner product of $H_{1}$ and $H_{2}$, respectively, where $\alpha=\left(a_{1}, a_{2}\right), \beta=\left(b_{1}, b_{2}\right) \in H_{1}, \gamma=\left(c_{1}, c_{2}, c_{3}\right), \eta=\left(d_{1}, d_{2}, d_{3}\right) \in H_{2}$. Let $A \alpha=\left(a_{2}, a_{1}, a_{1}-a_{2}\right)$ for $\alpha=\left(a_{1}, a_{2}\right) \in H_{1}$ and $B \gamma=\left(c_{2}+c_{3}, c_{1}-c_{3}\right)$ for $\gamma=\left(c_{1}, c_{2}, c_{3}\right) \in H_{2}$. Obviously, $B$ is an adjoint operator of $A$.

Let $K$ be a closed convex subset of a real Hilbert space $H$. For each point $x \in H$, there exists a unique nearest point in $K$, denoted by $P_{K} x$, such that $\left\|x-P_{K} x\right\| \leq\|x-y\| \forall y \in K$. The mapping $P_{K}$ is called the metric projection from $H$ onto $K$. It is well known that $P_{K}$ has the following characteristics:

(i) $\left\langle x-y, P_{K} x-P_{K} y\right\rangle \geq\left\|P_{K} x-P_{K} y\right\|^{2}$ for every $x, y \in H$;

(ii) for $x \in H$ and $z \in K, z=P_{K}(x) \Leftrightarrow\langle x-z, z-y\rangle \geq 0, \forall y \in K$;

(iii) for $x \in H$ and $y \in K$,

$$
\left\|y-P_{K}(x)\right\|^{2}+\left\|x-P_{K}(x)\right\|^{2} \leq\|x-y\|^{2} .
$$

Lemma 2.1 (see [1]) Let $K$ be a nonempty closed convex subset of $H$ and $F$ be a bi-function of $K \times K$ into $\mathbb{R}$ satisfying the following conditions:

(A1) $F(x, x)=0$ for all $x \in K$;

(A2) $F$ is monotone, that is, $F(x, y)+F(y, x) \leq 0$ for all $x, y \in K$;

(A3) for each $x, y, z \in K, \lim \sup _{t \downarrow 0} F(t z+(1-t) x, y) \leq F(x, y)$;

(A4) for each $x \in K, y \mapsto F(x, y)$ is convex and lower semi-continuous.

Let $r>0$ and $x \in H$. Then there exists $z \in K$ such that $F(z, y)+\frac{1}{r}\langle y-z, z-x\rangle \geq 0$ for all $y \in K$.

Lemma 2.2 (see [12]) Let $K$ be a nonempty closed convex subset of $H$ and let $F$ be a bifunction of $K \times K$ into $\mathbb{R}$ satisfying (A1)-(A4). For $r>0$, define a mapping $T_{r}^{F}: H \rightarrow K$ as follows:

$$
T_{r}^{F}(x)=\left\{z \in K: F(z, y)+\frac{1}{r}\langle y-z, z-x\rangle \geq 0, \forall y \in K\right\}
$$


for all $x \in H$. Then the following hold:

(i) $T_{r}^{F}$ is single-valued and $\mathcal{F}\left(T_{r}^{F}\right)=E P(F)$ for $\forall r>0$ and $E P(F)$ is closed and convex;

(ii) $T_{r}^{F}$ is firmly non-expansive, that is, for any $x, y \in H$, $\left\|T_{r}^{F} x-T_{r}^{F} y\right\|^{2} \leq\left\langle T_{r}^{F} x-T_{r}^{F} y, x-y\right\rangle$.

Lemma 2.3 (see, e.g., [6]) Let $H$ be a real Hilbert space. Then the following hold:

(a) $\|x+y\|^{2} \leq\|y\|^{2}+2\langle x, x+y\rangle$;

(b) $\|x-y\|^{2}=\|x\|^{2}+\|y\|^{2}-2\langle x, y\rangle$ for all $x, y \in H$;

(c) $\|\alpha x+(1-\alpha) y\|^{2}=\alpha\|x\|^{2}+(1-\alpha)\|y\|^{2}-\alpha(1-\alpha)\|x-y\|^{2}$ for all $x, y \in H$ and $\alpha \in[0,1]$.

Lemma 2.4 Let $F_{r}^{F}$ be the same as in Lemma 2.2. If $\mathcal{F}\left(T_{r}^{F}\right)=E P(F) \neq \emptyset$, then for any $x \in H$ and $x^{*} \in \mathcal{F}\left(T_{r}^{F}\right),\left\|T_{r}^{F} x-x\right\|^{2} \leq\left\|x-x^{*}\right\|^{2}-\left\|T_{r}^{F} x-x^{*}\right\|^{2}$.

Proof By (ii) of Lemma 2.2 and (b) of Lemma 2.3,

$$
\left\|T_{r}^{F} x-x^{*}\right\|^{2} \leq\left\langle T_{r}^{F} x-x^{*}, x-x^{*}\right\rangle=\frac{1}{2}\left(\left\|T_{r}^{F} x-x^{*}\right\|^{2}+\left\|x-x^{*}\right\|^{2}-\left\|T_{r}^{F} x-x\right\|^{2}\right),
$$

which shows that $\left\|T_{r}^{F} x-x\right\|^{2} \leq\left\|x-x^{*}\right\|^{2}-\left\|T_{r}^{F} x-x^{*}\right\|^{2}$.

Lemma $2.5([10,11])$ Let the mapping $T_{r}^{F}$ be defined as in Lemma 2.2. Then, for $r, s>0$ and $x, y \in H$,

$$
\left\|T_{r}^{F}(x)-T_{s}^{F}(y)\right\| \leq\|x-y\|+\frac{|s-r|}{s}\left\|T_{s}^{F}(y)-y\right\| .
$$

In particular, $\left\|T_{r}^{F}(x)-T_{r}^{F}(y)\right\| \leq\|x-y\|$ for any $r>0$ and $x, y \in H$, that is, $T_{r}^{F}$ is nonexpansive for any $r>0$.

Remark 2.1 In fact, Lemma 2.5 is motivated by a proof of [5, Theorem 3.2]. In order to the sake of convenience for proving, we restated the fact and gave its proof in Lemma 2.5 $[10,11]$.

Lemma 2.6 ([13]) Let $\left\{a_{n}\right\}$ be a nonnegative real sequence satisfying the following condition:

$$
a_{n+1} \leq\left(1-\lambda_{n}\right) a_{n}+\lambda_{n} b_{n}, \quad \forall n \geq n_{0},
$$

where $n_{0}$ is some nonnegative integer, $\left\{\lambda_{n}\right\}$ is a sequence in $(0,1)$ and $\left\{b_{n}\right\}$ is a sequence in $\mathbf{R}$ such that

(i) $\sum_{n=0}^{\infty} \lambda_{n}=\infty$;

(ii) $\limsup _{n \rightarrow \infty} b_{n} \leq 0$ or $\sum_{n=0}^{\infty} \lambda_{n} b_{n}$ is convergent. Then $\lim _{n \rightarrow \infty} a_{n}=0$.

Lemma 2.7 ([14]) Let $\left\{x_{n}\right\}$ and $\left\{y_{n}\right\}$ be bounded sequences in a Banach space $E$ and let $\left\{\beta_{n}\right\}$ be a sequence in $[0,1]$ with $0<\liminf \beta_{n} \leq \lim \sup \beta_{n}<1$. Suppose $x_{n+1}=\beta_{n} y_{n}+\left(1-\beta_{n}\right) x_{n}$ for all integers $n \geq 0$ and $\lim \sup _{n \rightarrow \infty}\left(\left\|y_{n+1}-y_{n}\right\|-\left\|x_{n+1}-x_{n}\right\|\right) \leq 0$, then $\lim _{n \rightarrow \infty}\left\|y_{n}-x_{n}\right\|=0$. 


\section{Weak convergence iterative algorithms for (HSP)}

In this section, we will introduce some weak convergence iterative algorithms for the hybrid split problem.

Theorem 3.1 Let $H_{1}$ and $H_{2}$ be two real Hilbert spaces. Let $C \subset H_{1}$ and $K \subset H_{2}$ be two nonempty closed convex sets. Let $T: C \rightarrow C$ and $S: K \rightarrow K$ be non-expansive mappings and $f: C \times C \rightarrow \mathbb{R}$ and $g: K \times K \rightarrow \mathbb{R}$ be bi-functions satisfying the conditions (A1)-(A4). Let $A: H_{1} \rightarrow H_{2}$ be a bounded linear operator with its adjoint B. Let $x_{1} \in C,\left\{x_{n}\right\}$ and $\left\{u_{n}\right\}$ be sequences generated by

$$
\left\{\begin{array}{l}
u_{n}=T_{r_{n}}^{f} x_{n}, \\
y_{n}=(1-\alpha) u_{n}+\alpha T u_{n}, \\
w_{n}=T_{r_{n}}^{g} A y_{n}, \\
x_{n+1}=P_{C}\left(y_{n}+\xi B\left(S w_{n}-A y_{n}\right)\right), \quad \forall n \in \mathbb{N},
\end{array}\right.
$$

where $\alpha \in(0,1), \xi \in\left(0, \frac{1}{\|B\|^{2}}\right)$ and $\left\{r_{n}\right\} \subset(0,+\infty)$ with $\liminf _{n \rightarrow+\infty} r_{n}>0, P_{C}$ is a projection operator from $H_{1}$ into C. Suppose that $\Omega=\{p \in \mathcal{F}(T) \cap E P(f): A p \in \mathcal{F}(S) \cap E P(g)\} \neq \emptyset$, then $x_{n}, u_{n} \rightarrow q \in \Omega$ and $w_{n} \rightarrow A q \in \mathcal{F}(S) \cap E P(g)$.

Proof Let $p \in \Omega$, the following several inequalities can be proved easily:

$$
\left\|y_{n}-p\right\| \leq\left\|u_{n}-p\right\| \leq\left\|x_{n}-p\right\|, \quad\left\|w_{n}-A p\right\| \leq\left\|A y_{n}-A p\right\| .
$$

By Lemma 2.4, $\left\|T_{r_{n}}^{g} A y_{n}-A y_{n}\right\|^{2} \leq\left\|A y_{n}-A p\right\|^{2}-\left\|T_{r_{n}}^{g} A y_{n}-A p\right\|^{2}$, hence

$$
\begin{aligned}
\left\|S w_{n}-A p\right\|^{2} & =\left\|S T_{r_{n}}^{g} A y_{n}-A p\right\|^{2} \leq\left\|T_{r_{n}}^{g} A y_{n}-A p\right\|^{2} \\
& \leq\left\|A y_{n}-A p\right\|^{2}-\left\|T_{r_{n}}^{g} A y_{n}-A y_{n}\right\|^{2} .
\end{aligned}
$$

By (b) of Lemma 2.3 and (3.3), for each $n \in \mathbb{N}$, we have

$$
\begin{aligned}
2 \xi\left\langle y_{n}-p, B\left(S T_{r_{n}}^{g}-I\right) A y_{n}\right\rangle \\
=2 \xi\left\langle A\left(y_{n}-p\right)+\left(S T_{r_{n}}^{g}-I\right) A y_{n}-\left(S T_{r_{n}}^{g}-I\right) A y_{n},\left(S T_{r_{n}}^{g}-I\right) A y_{n}\right\rangle \\
=2 \xi\left(\frac{1}{2}\left\|S T_{r_{n}}^{g} A y_{n}-A p\right\|^{2}+\frac{1}{2}\left\|\left(S T_{r_{n}}^{g}-I\right) A y_{n}\right\|^{2}\right. \\
\left.\quad-\frac{1}{2}\left\|A y_{n}-A p\right\|^{2}-\left\|\left(S T_{r_{n}}^{g}-I\right) A y_{n}\right\|^{2}\right) \\
\leq 2 \xi\left(-\frac{1}{2}\left\|T_{r_{n}}^{g} A y_{n}-A y_{n}\right\|^{2}+\frac{1}{2}\left\|\left(S T_{r_{n}}^{g}-I\right) A y_{n}\right\|^{2}-\left\|\left(S T_{r_{n}}^{g}-I\right) A y_{n}\right\|^{2}\right) \\
=-\xi\left\|\left(S T_{r_{n}}^{g}-I\right) A y_{n}\right\|^{2}-\xi\left\|T_{r_{n}}^{g} A y_{n}-A y_{n}\right\|^{2} .
\end{aligned}
$$

On the other hand, $\left\|B\left(S T_{r_{n}}^{g}-I\right) A y_{n}\right\|^{2} \leq\|B\|^{2}\left\|\left(S T_{r_{n}}^{g}-I\right) A y_{n}\right\|^{2}$, so from (3.1)-(3.4), we have

$$
\begin{aligned}
\left\|x_{n+1}-p\right\|^{2} & =\left\|P_{C}\left(y_{n}+\xi B\left(S T_{r_{n}}^{g}-I\right) A y_{n}\right)-p\right\|^{2} \leq\left\|y_{n}+\xi B\left(S T_{r_{n}}^{g}-I\right) A y_{n}-p\right\|^{2} \\
& =\left\|y_{n}-p\right\|^{2}+\left\|\xi B\left(S T_{r_{n}}^{g}-I\right) A y_{n}\right\|^{2}+2 \xi\left\langle y_{n}-p, B\left(S T_{r_{n}}^{g}-I\right) A y_{n}\right\rangle
\end{aligned}
$$




$$
\begin{aligned}
\leq & \left\|y_{n}-p\right\|^{2}+\xi^{2}\|B\|^{2}\left\|\left(S T_{r_{n}}^{g}-I\right) A y_{n}\right\|^{2}-\xi\left\|\left(S T_{r_{n}}^{g}-I\right) A y_{n}\right\|^{2} \\
& -\xi\left\|\left(T_{r_{n}}^{g}-I\right) A y_{n}\right\|^{2} \\
= & \left\|y_{n}-p\right\|^{2}-\xi\left(1-\xi\|B\|^{2}\right)\left\|\left(S T_{r_{n}}^{f}-I\right) A y_{n}\right\|^{2}-\xi\left\|\left(T_{r_{n}}^{g}-I\right) A y_{n}\right\|^{2} \\
\leq & \left\|x_{n}-p\right\|^{2}-\xi\left(1-\xi\|B\|^{2}\right)\left\|\left(S T_{r_{n}}^{f}-I\right) A y_{n}\right\|^{2}-\xi\left\|\left(T_{r_{n}}^{g}-I\right) A y_{n}\right\|^{2} .
\end{aligned}
$$

Since $\xi \in\left(0, \frac{1}{\|B\|^{2}}\right), \xi\left(1-\xi\|B\|^{2}\right)>0$, by (3.2) and (3.5), we have

$$
\left\|x_{n+1}-p\right\| \leq\left\|y_{n}-p\right\| \leq\left\|u_{n}-p\right\| \leq\left\|x_{n}-p\right\|
$$

and

$$
\xi\left(1-\xi\|B\|^{2}\right)\left\|\left(S T_{r_{n}}^{g}-I\right) A y_{n}\right\|^{2}+\xi\left\|\left(T_{r_{n}}^{g}-I\right) A y_{n}\right\|^{2} \leq\left\|x_{n}-p\right\|^{2}-\left\|x_{n+1}-p\right\|^{2} .
$$

The inequality (3.6) implies $\lim _{n \rightarrow \infty}\left\|x_{n}-p\right\|$ exists. Further, from (3.6) and (3.7), we get

$$
\begin{aligned}
& \lim _{n \rightarrow \infty}\left\|x_{n}-p\right\|=\lim _{n \rightarrow \infty}\left\|y_{n}-p\right\|=\lim _{n \rightarrow \infty}\left\|u_{n}-p\right\|, \\
& \lim _{n \rightarrow \infty}\left\|\left(S T_{r_{n}}^{g}-I\right) A y_{n}\right\|=\lim _{n \rightarrow \infty}\left\|\left(T_{r_{n}}^{g}-I\right) A y_{n}\right\|=\lim _{n \rightarrow \infty}\left\|w_{n}-A y_{n}\right\|=0 .
\end{aligned}
$$

The inequality (3.8) also implies that

$$
\lim _{n \rightarrow \infty}\left\|S w_{n}-w_{n}\right\|=0
$$

Using Lemma 2.4 and (3.8), we have

$$
\begin{aligned}
\left\|u_{n}-x_{n}\right\|^{2} & =\left\|T_{r_{n}}^{f} x_{n}-x_{n}\right\|^{2} \leq\left\|x_{n}-p\right\|^{2}-\left\|T_{r_{n}}^{f} x_{n}-p\right\|^{2} \\
& =\left\|x_{n}-p\right\|^{2}-\left\|u_{n}-p\right\|^{2} \rightarrow 0 .
\end{aligned}
$$

Notice that

$$
\begin{aligned}
\left\|y_{n}-p\right\|^{2} & =(1-\alpha)\left\|u_{n}-p\right\|^{2}+\alpha\left\|T u_{n}-p\right\|^{2}-\alpha(1-\alpha)\left\|T u_{n}-u_{n}\right\|^{2} \\
& \leq\left\|u_{n}-p\right\|^{2}-\alpha(1-\alpha)\left\|T u_{n}-u_{n}\right\|^{2}
\end{aligned}
$$

hence,

$$
\lim _{n \rightarrow \infty}\left\|T u_{n}-u_{n}\right\|=0 .
$$

From (3.10) and (3.11), we also have

$$
\begin{aligned}
\left\|y_{n}-x_{n}\right\| & \leq\left\|y_{n}-u_{n}\right\|+\left\|u_{n}-x_{n}\right\| \\
& =\alpha\left\|T u_{n}-u_{n}\right\|+\left\|u_{n}-x_{n}\right\| \rightarrow 0 \text { as } n \rightarrow \infty .
\end{aligned}
$$

The existence of $\lim _{n \rightarrow \infty}\left\|x_{n}-p\right\|$ implies that $\left\{x_{n}\right\}$ is bounded, hence $\left\{x_{n}\right\}$ has a weak convergence subsequence $\left\{x_{n_{j}}\right\}$. Assume that $x_{n_{j}} \rightarrow q$ for some $q \in C$, then $y_{n_{j}} \rightarrow q, A y_{n_{j}} \rightarrow$ $A q \in K$ and $w_{n_{j}}=T_{r_{n_{j}}}^{g} A y_{n_{j}} \rightarrow A q$ by (3.12) and (3.8). 
We say $q \in \Omega$, in other words, $q \in \mathcal{F}(T) \cap E P(f)$ and $A q \in \mathcal{F}(S) \cap E P(g)$. By (3.10), we also obtain $u_{n_{j}} \rightarrow q$. If $T q \neq q$, then, by Opial's condition and (3.11), we get

$$
\begin{aligned}
\liminf _{j \rightarrow \infty}\left\|u_{n_{j}}-q\right\| & <\liminf _{j \rightarrow \infty}\left\|u_{n_{j}}-T q\right\| \\
& \leq \liminf _{j \rightarrow \infty}\left\|u_{n_{j}}-T u_{n_{j}}+T u_{n_{j}}-T q\right\| \\
& \leq \liminf _{j \rightarrow \infty}\left\|u_{n_{j}}-q\right\|,
\end{aligned}
$$

which is a contradiction. Hence $T q=q$ or $q \in \mathcal{F}(T)$. On the other hand, from Lemma 2.2, we know $E P(f)=\mathcal{F}\left(T_{r}^{f}\right)$ for any $r>0$. Hence, if $T_{r}^{f} q \neq q$ for $r>0$, then by Opial's condition and (3.10) and Lemma 2.5, we have

$$
\begin{aligned}
\liminf _{j \rightarrow \infty}\left\|x_{n_{j}}-q\right\| & <\liminf _{j \rightarrow \infty}\left\|x_{n_{j}}-T_{r}^{f} q\right\| \\
& =\liminf _{j \rightarrow \infty}\left\|x_{n_{j}}-T_{r_{n_{j}}}^{f} x_{n_{j}}+T_{r_{n_{j}}}^{f} x_{n_{j}}-T_{r}^{f} q\right\| \\
& \leq \liminf _{j \rightarrow \infty}\left\{\left\|x_{n_{j}}-T_{r_{n_{j}}}^{f} x_{n_{j}}\right\|+\left\|T_{r}^{f} q-T_{r_{n_{j}}}^{f} x_{n_{j}}\right\|\right\} \\
& \leq \liminf _{j \rightarrow \infty}\left\{\left\|x_{n_{j}}-T_{r_{n_{j}}}^{f} x_{n_{j}}\right\|+\left\|x_{n_{j}}-q\right\|+\frac{\left|r-r_{n_{j}}\right|}{r_{n_{j}}}\left\|T_{r_{n_{j}}}^{f} x_{n_{j}}-x_{n_{j}}\right\|\right\} \\
& =\liminf _{j \rightarrow \infty}\left\|x_{n_{j}}-q\right\|,
\end{aligned}
$$

which is also a contradiction. So, for each $r>0, T_{r}^{f} q=q$, namely $q \in E P(f)$. Thus, we have proved $q \in \mathcal{F}(T) \cap E P(f)$. Similarly, we can also prove $A q \in \mathcal{F}(S) \cap E P(g)$. Hence, $q \in \Omega$.

Finally, we prove $\left\{x_{n}\right\}$ converges weakly to $q \in \Omega$. Otherwise, if there exists another subsequence of $\left\{x_{n}\right\}$, which is denoted by $\left\{x_{n_{l}}\right\}$, such that $x_{n_{l}} \rightarrow \bar{x} \in \Omega$ with $\bar{x} \neq q$, then by Opial's condition,

$$
\liminf _{l \rightarrow \infty}\left\|x_{n_{l}}-\bar{x}\right\|<\liminf _{l \rightarrow \infty}\left\|x_{n_{l}}-q\right\|=\liminf _{j \rightarrow \infty}\left\|x_{n_{j}}-q\right\|<\liminf _{l \rightarrow \infty}\left\|x_{n_{l}}-\bar{x}\right\| .
$$

This is a contradiction. Hence $\left\{x_{n}\right\}$ converges weakly to an element $q \in \Omega$. Together with $\left\|u_{n}-x_{n}\right\| \rightarrow 0$ (see (3.10)), we also get $u_{n} \rightarrow q$.

Finally, we prove $\left\{w_{n}=T_{r_{n}}^{g} A y_{n}\right\}$ converges weakly to $A q \in \mathcal{F}(S) \cap E P(g)$. From (3.12), we have $y_{n} \rightarrow q$, so $A y_{n} \rightarrow A q$. Thus, from (3.8) we have $w_{n}=T_{r_{n}}^{g} A y_{n} \rightarrow A q \in \mathcal{F}(S) \cap E P(g)$. The proof is completed.

If $T=I$ or $S=I$, where $I$ denotes an identity operator, then the following corollaries follow from Theorem 3.1.

Corollary 3.1 Let $H_{1}$ and $H_{2}$ be two real Hilbert spaces. Let $C \subset H_{1}$ and $K \subset H_{2}$ be two nonempty closed convex sets. Let $S: K \rightarrow K$ be a non-expansive mapping and $f: C \times C \rightarrow \mathbb{R}$ and $g: K \times K \rightarrow \mathbb{R}$ be bi-functions satisfying the conditions (A1)-(A4). Let $A: H_{1} \rightarrow H_{2}$ be a bounded linear operator with its adjoint $B$. Let $x_{1} \in C,\left\{x_{n}\right\}$ and $\left\{u_{n}\right\}$ be sequences generated 
by

$$
\left\{\begin{array}{l}
u_{n}=T_{r_{n}}^{f} x_{n}, \\
w_{n}=T_{r_{n}}^{g} A u_{n}, \\
x_{n+1}=P_{C}\left(u_{n}+\xi B\left(S w_{n}-A u_{n}\right)\right), \quad \forall n \in \mathbb{N},
\end{array}\right.
$$

where $\xi \in\left(0, \frac{1}{\|B\|^{2}}\right)$ and $\left\{r_{n}\right\} \subset(0,+\infty)$ with $\liminf _{n \rightarrow+\infty} r_{n}>0, P_{C}$ is a projection operator from $H_{1}$ into $C$. Suppose that $\Omega=\{p \in E P(f): A p \in \mathcal{F}(S) \cap E P(g)\} \neq \emptyset$, then $x_{n}, u_{n} \rightarrow q \in \Omega$ and $w_{n} \rightarrow A q \in \mathcal{F}(S) \cap E P(g)$.

Corollary 3.2 Let $H_{1}$ and $H_{2}$ be two real Hilbert spaces. Let $C \subset H_{1}$ and $K \subset H_{2}$ be two nonempty closed convex sets. Let $T: C \rightarrow C$ be a non-expansive mapping and $f: C \times C \rightarrow$ $\mathbb{R}$ and $g: K \times K \rightarrow \mathbb{R}$ be bi-functions satisfying the conditions (A1)-(A4). Let $A: H_{1} \rightarrow H_{2}$ be a bounded linear operator with its adjoint $B$. Let $x_{1} \in C,\left\{x_{n}\right\}$ and $\left\{u_{n}\right\}$ be sequences generated by

$$
\left\{\begin{array}{l}
u_{n}=T_{r_{n}}^{f} x_{n}, \\
y_{n}=(1-\alpha) u_{n}+\alpha T u_{n}, \\
w_{n}=T_{r_{n}}^{g} A y_{n}, \\
x_{n+1}=P_{C}\left(y_{n}+\xi B\left(w_{n}-A y_{n}\right)\right), \quad \forall n \in \mathbb{N},
\end{array}\right.
$$

where $\alpha \in(0,1), \xi \in\left(0, \frac{1}{\|B\|^{2}}\right)$ and $\left\{r_{n}\right\} \subset(0,+\infty)$ with $\liminf _{n \rightarrow+\infty} r_{n}>0, P_{C}$ is a projection operator from $H_{1}$ into $C$. Suppose that $\Omega=\{p \in \mathcal{F}(T) \cap E P(f): A p \in E P(g)\} \neq \emptyset$, then $x_{n}, u_{n} \rightarrow q \in \Omega$ and $w_{n} \rightarrow A q \in E P(g)$.

Corollary 3.3 Let $C \subset H_{1}$ and $K \subset H_{2}$ be two nonempty closed convex sets. Letf $: C \times C \rightarrow$ $\mathbb{R}$ and $g: K \times K \rightarrow \mathbb{R}$ be bi-functions satisfying the conditions (A1)-(A4). Let $A: H_{1} \rightarrow H_{2}$ be a bounded linear operator with its adjoint $B$. Let $x_{1} \in C,\left\{x_{n}\right\}$ and $\left\{u_{n}\right\}$ be sequences generated by

$$
\left\{\begin{array}{l}
u_{n}=T_{r_{n}}^{f} x_{n}, \\
w_{n}=T_{r_{n}}^{g} A u_{n}, \\
x_{n+1}=P_{C}\left(u_{n}+\xi B\left(w_{n}-A u_{n}\right)\right), \quad \forall n \in \mathbb{N},
\end{array}\right.
$$

where $\xi \in\left(0, \frac{1}{\|B\|^{2}}\right)$ and $\left\{r_{n}\right\} \subset(0,+\infty)$ with $\liminf _{n \rightarrow+\infty} r_{n}>0, P_{C}$ is a projection operator from $H_{1}$ into C. Suppose that $\Omega=\{p \in E P(f): A p \in E P(g)\} \neq \emptyset$, then $x_{n}, u_{n} \rightarrow q \in \Omega$ and $w_{n} \rightarrow A q \in E P(g)$.

\section{Strong convergence iterative algorithms for (HSP)}

In this section, we introduce two strong convergence algorithms for (HSP); see Theorem 4.1 and Theorem 4.2.

Theorem 4.1 Let $H_{1}$ and $H_{2}$ be two real Hilbert spaces. Let $C \subset H_{1}$ and $K \subset H_{2}$ be two nonempty closed convex sets. Let $T: C \rightarrow C$ and $S: K \rightarrow K$ be non-expansive mappings 
and $f: C \times C \rightarrow \mathbb{R}$ and $g: K \times K \rightarrow \mathbb{R}$ be bi-functions satisfying the conditions (A1)-(A4). Let $A: H_{1} \rightarrow H_{2}$ be a bounded linear operator with its adjoint $B$. Let $x_{1} \in C_{1}:=C,\left\{x_{n}\right\}$ and $\left\{u_{n}\right\}$ be sequences generated by

$$
\left\{\begin{array}{l}
u_{n}=T_{r_{n}}^{f} x_{n}, \\
y_{n}=(1-\alpha) u_{n}+\alpha T u_{n}, \\
w_{n}=T_{r_{n}}^{g} A y_{n}, \\
z_{n}=P_{C}\left(y_{n}+\xi B\left(S w_{n}-A y_{n}\right)\right), \\
C_{n+1}=\left\{v \in C_{n}:\left\|z_{n}-v\right\| \leq\left\|y_{n}-v\right\| \leq\left\|x_{n}-v\right\|\right\}, \\
x_{n+1}=P_{C_{n+1}}\left(x_{1}\right), \quad n \in \mathbb{N},
\end{array}\right.
$$

where $\alpha \in(0,1), \xi \in\left(0, \frac{1}{\|B\|^{2}}\right)$ and $\left\{r_{n}\right\} \subset(0,+\infty)$ with $\liminf _{n \rightarrow+\infty} r_{n}>0, P_{C}$ is a projection operator from $H_{1}$ into C. Suppose that $\Omega=\{p \in \mathcal{F}(T) \cap E P(f): A p \in \mathcal{F}(S) \cap E P(g)\} \neq \emptyset$, then $x_{n}, u_{n} \rightarrow q \in \Omega$ and $w_{n} \rightarrow A q \in \mathcal{F}(S) \cap E P(g)$.

Proof We claim that $C_{n}$ is a nonempty closed convex set for $n \in \mathbb{N}$. In fact, let $p \in \Omega$, it follows from (3.4) that

$$
2 \xi\left\langle y_{n}-p, B\left(S w_{n}-A y_{n}\right)\right\rangle \leq-\xi\left\|\left(T_{r_{n}}^{g}-I\right) A x_{n}\right\|^{2}-\xi\left\|S w_{n}-A y_{n}\right\|^{2}
$$

By (3.2), (4.1) and (4.2), we obtain

$$
\begin{aligned}
\left\|z_{n}-p\right\|^{2} \leq & \left\|y_{n}+\xi B\left(S w_{n}-A y_{n}\right)-p\right\|^{2} \\
= & \left\|y_{n}-p\right\|^{2}+\left\|\xi B\left(S w_{n}-A y_{n}\right)\right\|^{2}+2 \xi\left(y_{n}-p, B\left(S w_{n}-A y_{n}\right)\right\rangle \\
\leq & \left\|y_{n}-p\right\|^{2}+\xi{ }^{2}\|B\|^{2}\left\|S w_{n}-A y_{n}\right\|^{2}-\xi\left\|\left(T_{r_{n}}^{g}-I\right) A y_{n}\right\|^{2}-\xi\left\|S w_{n}-A y_{n}\right\|^{2} \\
= & \left\|y_{n}-p\right\|^{2}-\xi\left(1-\xi\|B\|^{2}\right)\left\|\left(S T_{r_{n}}^{g}-I\right) A y_{n}\right\|^{2}-\xi\left\|\left(T_{r_{n}}^{g}-I\right) A y_{n}\right\|^{2} \\
\leq & \left\|u_{n}-p\right\|^{2}-(1-\alpha) \alpha\left\|u_{n}-T u_{n}\right\|^{2} \\
& -\xi\left(1-\xi\|B\|^{2}\right)\left\|\left(S T_{r_{n}}^{g}-I\right) A y_{n}\right\|^{2}-\xi\left\|\left(T_{r_{n}}^{g}-I\right) A y_{n}\right\|^{2} \\
\leq & \left\|x_{n}-p\right\|^{2}-\xi\left(1-\xi\|B\|^{2}\right)\left\|\left(S T_{r_{n}}^{g}-I\right) A y_{n}\right\|^{2} \\
& -\xi\left\|\left(T_{r_{n}}^{g}-I\right) A y_{n}\right\|^{2}-(1-\alpha) \alpha\left\|u_{n}-T u_{n}\right\|^{2} .
\end{aligned}
$$

Notice $\xi \in\left(0, \frac{1}{\|B\|^{2}}\right), \xi\left(1-\xi\|B\|^{2}\right)>0$. It follows from (4.3) that

$$
\left\|z_{n}-p\right\| \leq\left\|y_{n}-p\right\| \leq\left\|u_{n}-p\right\| \leq\left\|x_{n}-p\right\| \quad \text { for all } n \in \mathbb{N},
$$

hence $p \in C_{n}$, which yields that $\Omega \subset C_{n}$ and $C_{n} \neq \emptyset$ for $n \in \mathbb{N}$.

It is not hard to verify that $C_{n}$ is closed for $n \in \mathbb{N}$, so it suffices to verify $C_{n}$ is convex for $n \in \mathbb{N}$. Indeed, let $w_{1}, w_{2} \in C_{n+1}$ and $\gamma \in[0,1]$, we have

$$
\begin{aligned}
& \left\|z_{n}-\left(\gamma w_{1}+(1-\gamma) w_{2}\right)\right\|^{2} \\
& \quad=\left\|\gamma\left(z_{n}-w_{1}\right)+(1-\gamma)\left(z_{n}-w_{2}\right)\right\|^{2}
\end{aligned}
$$




$$
\begin{aligned}
& =\gamma\left\|z_{n}-w_{1}\right\|^{2}+(1-\gamma)\left\|z_{n}-w_{2}\right\|^{2}-\gamma(1-\gamma)\left\|w_{1}-w_{2}\right\|^{2} \\
& \leq \gamma\left\|y_{n}-w_{1}\right\|^{2}+(1-\gamma)\left\|y_{n}-w_{2}\right\|^{2}-\gamma(1-\gamma)\left\|w_{1}-w_{2}\right\|^{2} \\
& =\left\|y_{n}-\left(\gamma w_{1}+(1-\gamma) w_{2}\right)\right\|^{2},
\end{aligned}
$$

namely $\left\|z_{n}-\left(\gamma w_{1}+(1-\gamma) w_{2}\right)\right\| \leq\left\|y_{n}-\left(\gamma w_{1}+(1-\gamma) w_{2}\right)\right\|$. Similarly, $\| y_{n}-\left(\gamma w_{1}+(1-\right.$ $\left.\gamma) w_{2}\right)\|\leq\| x_{n}-\left(\gamma w_{1}+(1-\gamma) w_{2}\right) \|$, which implies $\gamma w_{1}+(1-\gamma) w_{2} \in C_{n+1}$ and $C_{n+1}$ is a convex set, $n \in \mathbb{N}$.

Notice that $C_{n+1} \subset C_{n}$ and $x_{n+1}=P_{C_{n+1}}\left(x_{1}\right) \subset C_{n}$, then $\left\|x_{n+1}-x_{1}\right\| \leq\left\|x_{n}-x_{1}\right\|$ for $n>1$. It follows that $\lim _{n \rightarrow \infty}\left\|x_{n}-x_{1}\right\|$ exists. Hence $\left\{x_{n}\right\}$ is bounded, which yields that $\left\{z_{n}\right\}$ and $\left\{y_{n}\right\}$ are bounded. For some $k, n \in \mathbb{N}$ with $k>n>1$, from $x_{k}=P_{C_{k}}\left(x_{1}\right) \subset C_{n}$ and (2.1), we have

$$
\begin{aligned}
\left\|x_{n}-x_{k}\right\|^{2}+\left\|x_{1}-x_{k}\right\|^{2} & =\left\|x_{n}-P_{C_{k}}\left(x_{1}\right)\right\|^{2}+\left\|x_{1}-P_{C_{k}}\left(x_{1}\right)\right\|^{2} \\
& \leq\left\|x_{n}-x_{1}\right\|^{2} .
\end{aligned}
$$

By $\lim _{n \rightarrow \infty}\left\|x_{n}-x_{1}\right\|$ exists and (4.4), we have $\lim _{n \rightarrow \infty}\left\|x_{n}-x_{k}\right\|=0$, so $\left\{x_{n}\right\}$ is a Cauchy sequence.

Let $x_{n} \rightarrow q$, then $q \in \Omega$. Firstly, by $x_{n+1}=P_{C_{n+1}}\left(x_{1}\right) \in C_{n+1} \subset C_{n}$, from (4.1) we have

$$
\begin{aligned}
& \left\|z_{n}-x_{n}\right\| \leq\left\|z_{n}-x_{n+1}\right\|+\left\|x_{n+1}-x_{n}\right\| \leq 2\left\|x_{n+1}-x_{n}\right\| \rightarrow 0, \\
& \left\|y_{n}-x_{n}\right\| \leq\left\|y_{n}-x_{n+1}\right\|+\left\|x_{n+1}-x_{n}\right\| \leq 2\left\|x_{n+1}-x_{n}\right\| \rightarrow 0 .
\end{aligned}
$$

Setting $\rho=\xi\left(1-\xi\|B\|^{2}\right)$, by (4.3) again, we have

$$
\begin{aligned}
& \rho\left\|\left(S T_{r_{n}}^{g}-I\right) A y_{n}\right\|^{2}+\xi\left\|\left(T_{r_{n}}^{g}-I\right) A y_{n}\right\|^{2}+(1-\alpha) \alpha\left\|u_{n}-T u_{n}\right\|^{2} \\
& \quad \leq\left\|x_{n}-p\right\|^{2}-\left\|z_{n}-p\right\|^{2} \leq\left\|x_{n}-z_{n}\right\|\left\{\left\|x_{n}-p\right\|+\left\|z_{n}-p\right\|\right\} \rightarrow 0 .
\end{aligned}
$$

So,

$$
\begin{aligned}
& \lim _{n \rightarrow \infty}\left\|T u_{n}-u_{n}\right\|=0, \quad \lim _{n \rightarrow \infty}\left\|w_{n}-A y_{n}\right\|=\lim _{n \rightarrow \infty}\left\|\left(T_{r_{n}}^{g}-I\right) A y_{n}\right\|=0, \\
& \lim _{n \rightarrow \infty}\left\|S w_{n}-A y_{n}\right\|=\lim _{n \rightarrow \infty}\left\|\left(S T_{r_{n}}^{g}-I\right) A y_{n}\right\|=0, \quad \lim _{n \rightarrow \infty}\left\|S w_{n}-w_{n}\right\|=0 .
\end{aligned}
$$

Notice that $\lim _{n \rightarrow \infty}\left\|T u_{n}-u_{n}\right\|=0$ and $\left\|y_{n}-u_{n}\right\|=\alpha\left\|T u_{n}-u_{n}\right\|$, so

$$
\lim _{n \rightarrow \infty}\left\|y_{n}-u_{n}\right\|=0
$$

Further, from (4.5) and (4.8),

$$
\lim _{n \rightarrow \infty}\left\|x_{n}-u_{n}\right\|=0
$$

Since $x_{n} \rightarrow q$, we have $u_{n} \rightarrow q$ by (4.9). Thus

$$
\|T q-q\| \leq\left\|T q-T u_{n}\right\|+\left\|T u_{n}-u_{n}\right\|+\left\|u_{n}-q\right\| \rightarrow 0,
$$


namely $T q=q$ and $q \in \mathcal{F}(T)$. On the other hand, for $r>0$, by Lemma 2.5, we have

$$
\begin{aligned}
\left\|T_{r}^{f} q-q\right\| & \leq\left\|T_{r}^{f} q-T_{r_{n}}^{f} x_{n}+T_{r_{n}}^{f} x_{n}-x_{n}+x_{n}-q\right\| \\
& \leq\left\|x_{n}-q\right\|+\frac{\left|r_{n}-r\right|}{r_{n}}\left\|T_{r_{n}}^{f} x_{n}-x_{n}\right\|+\left\|T_{r_{n}}^{f} x_{n}-x_{n}\right\|+\left\|x_{n}-q\right\| \rightarrow 0,
\end{aligned}
$$

which yields $q \in \mathcal{F}\left(T_{r}^{f}\right)=E P(f)$. We have verified $q \in \mathcal{F}(T) \cap E P(f)$.

Next, we prove $A q \in \mathcal{F}(S) \cap E P(g)$. Since $x_{n} \rightarrow q$ and $x_{n}-y_{n} \rightarrow 0$ by (4.8) and (4.9) and $w_{n}-A y_{n} \rightarrow 0$ by (4.7), we have $y_{n} \rightarrow q$ and $A y_{n} \rightarrow A q$ and $w_{n} \rightarrow A q$. So,

$$
\|S A q-A q\| \leq\left\|S A q-S w_{n}\right\|+\left\|S w_{n}-w_{n}\right\|+\left\|w_{n}-A q\right\| \rightarrow 0,
$$

namely $S A q=A q$ and $A q \in \mathcal{F}(S)$. On the other hand, for $r>0$, by Lemma 2.5 again, we have

$$
\begin{aligned}
\left\|T_{r}^{g} A q-A q\right\| \leq & \left\|T_{r}^{g} A q-T_{r_{n}}^{g} A y_{n}+T_{r_{n}}^{g} A y_{n}-A y_{n}+A y_{n}-A q\right\| \\
\leq & \left\|A y_{n}-A q\right\|+\frac{\left|r_{n}-r\right|}{r_{n}}\left\|T_{r_{n}}^{g} A y_{n}-A y_{n}\right\| \\
& +\left\|T_{r_{n}}^{g} A y_{n}-A y_{n}\right\|+\left\|A y_{n}-A q\right\| \rightarrow 0,
\end{aligned}
$$

which implies that $A q \in \mathcal{F}\left(T_{r}^{g}\right)=E P(g)$. We have verified $A q \in \mathcal{F}(S) \cap E P(g)$.

So, we have obtained $q \in \Omega$ and $x_{n}, u_{n} \rightarrow q$ and $w_{n} \rightarrow A q$, the proof is completed.

If $T=I$ or $S=I$, where $I$ denotes an identity operator, then the following corollaries follow from Theorem 4.1.

Corollary 4.1 Let $H_{1}$ and $H_{2}$ be two real Hilbert spaces. Let $C \subset H_{1}$ and $K \subset H_{2}$ be two nonempty closed convex sets. Let $f: C \times C \rightarrow \mathbb{R}$ and $g: K \times K \rightarrow \mathbb{R}$ be bi-functions satisfying the conditions (A1)-(A4) and $S: K \rightarrow K$ be a non-expansive mapping. Let $A: H_{1} \rightarrow H_{2}$ be a bounded linear operator with its adjoint $B$. Let $x_{1} \in C_{1}:=C,\left\{x_{n}\right\}$ and $\left\{u_{n}\right\}$ be sequences generated by

$$
\left\{\begin{array}{l}
u_{n}=T_{r_{n}}^{f} x_{n}, \\
w_{n}=T_{r_{n}}^{g} A u_{n}, \\
z_{n}=P_{C}\left(u_{n}+\xi B\left(S w_{n}-A u_{n}\right)\right), \\
C_{n+1}=\left\{v \in C_{n}:\left\|z_{n}-v\right\| \leq\left\|u_{n}-v\right\| \leq\left\|x_{n}-v\right\|\right\}, \\
x_{n+1}=P_{C_{n+1}}\left(x_{1}\right), \quad n \in \mathbb{N},
\end{array}\right.
$$

where $\xi \in\left(0, \frac{1}{\|B\|^{2}}\right)$ and $\left\{r_{n}\right\} \subset(0,+\infty)$ with $\liminf _{n \rightarrow+\infty} r_{n}>0, P_{C}$ is a projection operator from $H_{1}$ into $C$. Suppose that $\Omega=\{p \in E P(f): A p \in \mathcal{F}(S) \cap E P(g)\} \neq \emptyset$, then $x_{n}, u_{n} \rightarrow q \in \Omega$ and $w_{n} \rightarrow A q \in \mathcal{F}(S) \cap E P(g)$.

Corollary 4.2 Let $H_{1}$ and $H_{2}$ be two real Hilbert spaces. Let $C \subset H_{1}$ and $K \subset H_{2}$ be two nonempty closed convex sets. Let $T: C \rightarrow C$ be a non-expansive mapping and $f: C \times C \rightarrow$ $\mathbb{R}$ and $g: K \times K \rightarrow \mathbb{R}$ be bi-functions satisfying the conditions (A1)-(A4). Let $A: H_{1} \rightarrow H_{2}$ 
be a bounded linear operator with its adjoint $B$. Let $x_{1} \in C_{1}:=C,\left\{x_{n}\right\}$ and $\left\{u_{n}\right\}$ be sequences generated by

$$
\left\{\begin{array}{l}
u_{n}=T_{r_{n}}^{f} x_{n} \\
y_{n}=(1-\alpha) u_{n}+\alpha T u_{n}, \\
w_{n}=T_{r_{n}}^{g} A y_{n} \\
z_{n}=P_{C}\left(y_{n}+\xi B\left(w_{n}-A y_{n}\right)\right), \\
C_{n+1}=\left\{v \in C_{n}:\left\|z_{n}-v\right\| \leq\left\|y_{n}-v\right\| \leq\left\|x_{n}-v\right\|\right\} \\
x_{n+1}=P_{C_{n+1}}\left(x_{1}\right), \quad n \in \mathbb{N},
\end{array}\right.
$$

where, $\alpha \in(0,1), \xi \in\left(0, \frac{1}{\|B\|^{2}}\right)$ and $\left\{r_{n}\right\} \subset(0,+\infty)$ with $\liminf _{n \rightarrow+\infty} r_{n}>0, P_{C}$ is a projection operator from $H_{1}$ into $C$. Suppose that $\Omega=\{p \in \mathcal{F}(T) \cap E P(f): A p \in E P(g)\} \neq \emptyset$, then $x_{n}, u_{n} \rightarrow q \in \Omega$ and $w_{n} \rightarrow A q \in E P(g)$.

Corollary 4.3 Let $H_{1}$ and $H_{2}$ be two real Hilbert spaces. Let $C \subset H_{1}$ and $K \subset H_{2}$ be two nonempty closed convex sets. Let $f: C \times C \rightarrow \mathbb{R}$ and $g: K \times K \rightarrow \mathbb{R}$ be bi-functions satisfying the conditions (A1)-(A4). Let $A: H_{1} \rightarrow H_{2}$ be a bounded linear operator with its adjoint $B$. Let $x_{1} \in C_{1}:=C,\left\{x_{n}\right\}$ and $\left\{u_{n}\right\}$ be sequences generated by

$$
\left\{\begin{array}{l}
u_{n}=T_{r_{n}}^{f} x_{n}, \quad w_{n}=T_{r_{n}}^{g} A u_{n}, \\
z_{n}=P_{C}\left(y_{n}+\xi B\left(w_{n}-A u_{n}\right)\right), \\
C_{n+1}=\left\{v \in C_{n}:\left\|z_{n}-v\right\| \leq\left\|u_{n}-v\right\| \leq\left\|x_{n}-v\right\|\right\}, \\
x_{n+1}=P_{C_{n+1}}\left(x_{1}\right), \quad n \in \mathbb{N},
\end{array}\right.
$$

where $\xi \in\left(0, \frac{1}{\|B\|^{2}}\right)$ and $\left\{r_{n}\right\} \subset(0,+\infty)$ with $\liminf _{n \rightarrow+\infty} r_{n}>0, P_{C}$ is a projection operator from $H_{1}$ into $C$. Suppose that $\Omega=\{p \in E P(f): A p \in E P(g)\} \neq \emptyset$, then $x_{n}, u_{n} \rightarrow q \in \Omega$ and $w_{n} \rightarrow A q \in E P(g)$.

It is well known that the viscosity iterative method is always applied to study the iterative solution for the fixed point problem of nonlinear operators, for example, $[5,6,8,15,16]$. Similarly, the viscosity iterative method can also be used to study the hybrid split problem (HSP). So, at the end of this paper, we introduce a viscosity iterative algorithm which can converge strongly to a solution of (HSP).

Theorem 4.2 Let $H_{1}$ and $H_{2}$ be two real Hilbert spaces. Let $C \subset H_{1}$ and $K \subset H_{2}$ be two nonempty closed convex sets. Let $h: C \rightarrow C$ be a $\alpha$-contraction mapping, $T: C \rightarrow C$ and $S: K \rightarrow K$ be non-expansive mappings and $f: C \times C \rightarrow \mathbb{R}$ and $g: K \times K \rightarrow \mathbb{R}$ be bifunctions satisfying the conditions (A1)-(A4). Let $A: H_{1} \rightarrow H_{2}$ be a bounded linear operator with its adjoint $B$. Let $x_{1} \in C,\left\{x_{n}\right\}$ and $\left\{u_{n}\right\}$ be sequences generated by

$$
\left\{\begin{array}{l}
u_{n}=T_{r_{n}}^{f} x_{n}, \\
w_{n}=T_{r_{n}}^{g} A u_{n}, \\
y_{n}=P_{C}\left(u_{n}+\xi B\left(S w_{n}-A u_{n}\right)\right), \\
z_{n}=(1-r) x_{n}+r T y_{n}, \\
x_{n+1}=\alpha_{n} h\left(x_{n}\right)+\left(1-\alpha_{n}\right) z_{n}, \quad n \in \mathbb{N},
\end{array}\right.
$$


where $r \in(0,1), \xi \in\left(0, \frac{1}{\|B\|^{2}}\right)$ and $\left\{r_{n}\right\} \subset(0,+\infty), P_{C}$ is a projection operator from $H_{1}$ into $C$, and the coefficients $\left\{\alpha_{n}\right\}$ and $\left\{r_{n}\right\}$ satisfy the following conditions:

(1) $\left\{\alpha_{n}\right\} \subset(0,1), \lim _{n \rightarrow \infty} \alpha_{n}=0, \sum_{n=1}^{\infty} \alpha_{n}=\infty$;

(2) $\liminf _{n \rightarrow+\infty} r_{n}>0, \lim _{n \rightarrow \infty}\left|r_{n+1}-r_{n}\right|=0$.

Suppose that $\Omega=\{p \in \mathcal{F}(T) \cap E P(f): A p \in \mathcal{F}(S) \cap E P(g)\} \neq \emptyset$, then $x_{n}, u_{n} \rightarrow q \in \Omega$ and $w_{n} \rightarrow A q \in \mathcal{F}(S) \cap E P(g)$, where $q=P_{\Omega} h(q)$.

Proof Let $p \in \Omega$. The following inequalities are easily verified:

$$
\left\|u_{n}-p\right\| \leq\left\|x_{n}-p\right\|, \quad\left\|w_{n}-A p\right\| \leq\left\|A u_{n}-A p\right\| .
$$

By Lemma 2.4,

$$
\begin{gathered}
\left\|u_{n}-p\right\|^{2} \leq\left\|x_{n}-p\right\|^{2}-\left\|T_{r_{n}}^{g} x_{n}-x_{n}\right\|^{2}=\left\|x_{n}-p\right\|^{2}-\left\|u_{n}-x_{n}\right\|^{2} ; \\
\left\|S w_{n}-A p\right\|^{2}=\left\|S T_{r_{n}}^{g} A u_{n}-A p\right\|^{2} \leq\left\|T_{r_{n}}^{g} A u_{n}-A p\right\|^{2} \\
\leq\left\|A u_{n}-A p\right\|^{2}-\left\|T_{r_{n}}^{g} A u_{n}-A u_{n}\right\|^{2} .
\end{gathered}
$$

From (4.10) and (4.12), we have

$$
\begin{aligned}
2 \xi & \left\langle u_{n}-p, B\left(S w_{n}-A u_{n}\right)\right\rangle \\
& =2 \xi\left\langle A\left(u_{n}-p\right)+S w_{n}-A u_{n}-\left(S w_{n}-A u_{n}\right), S w_{n}-A u_{n}\right\rangle \\
& =2 \xi\left(\frac{1}{2}\left\|S w_{n}-A p\right\|^{2}+\frac{1}{2}\left\|S w_{n}-A u_{n}\right\|^{2}-\frac{1}{2}\left\|A u_{n}-A p\right\|^{2}-\left\|S w_{n}-A u_{n}\right\|^{2}\right) \\
& \leq 2 \xi\left(-\frac{1}{2}\left\|T_{r_{n}}^{g} A u_{n}-A u_{n}\right\|^{2}-\frac{1}{2}\left\|S w_{n}-A u_{n}\right\|^{2}\right) \\
& =-\xi\left\|S w_{n}-A u_{n}\right\|^{2}-\xi\left\|T_{r_{n}}^{g} A u_{n}-A u_{n}\right\|^{2} \\
& =-\xi\left\|S w_{n}-A u_{n}\right\|^{2}-\xi\left\|w_{n}-A u_{n}\right\|^{2}
\end{aligned}
$$

and

$$
\begin{aligned}
\left\|y_{n}-p\right\|^{2} & =\| P_{C}\left(u_{n}+\xi B\left(S w_{n}-A u_{n}\right)-P_{C} p \|^{2}\right. \\
& \leq\left\|u_{n}-p+\xi B\left(S w_{n}-A u_{n}\right)\right\|^{2} \\
& =\left\|u_{n}-p\right\|^{2}+\left\|\xi B\left(S w_{n}-A u_{n}\right)\right\|^{2}+2 \xi\left\langle u_{n}-p, B\left(S w_{n}-A u_{n}\right)\right\rangle \\
& \leq\left\|u_{n}-p\right\|^{2}-\xi\left(1-\xi\|B\|^{2}\right)\left\|S w_{n}-A u_{n}\right\|^{2}-\xi\left\|T_{r_{n}}^{g} A u_{n}-A u_{n}\right\|^{2} \\
& \leq\left\|x_{n}-p\right\|^{2}-\xi\left(1-\xi\|B\|^{2}\right)\left\|S w_{n}-A u_{n}\right\|^{2}-\xi\left\|T_{r_{n}}^{g} A u_{n}-A u_{n}\right\|^{2} \\
& =\left\|x_{n}-p\right\|^{2}-\xi\left(1-\xi\|B\|^{2}\right)\left\|S w_{n}-A u_{n}\right\|^{2}-\xi\left\|w_{n}-A u_{n}\right\|^{2} .
\end{aligned}
$$

So, from (4.10)-(4.11) and (4.14), we have

$$
\left\|y_{n}-p\right\| \leq\left\|u_{n}-p\right\| \leq\left\|x_{n}-p\right\|, \quad\left\|z_{n}-p\right\| \leq\left\|x_{n}-p\right\| .
$$


We say $\left\{x_{n}\right\}$ is bounded. In fact, from (4.10) and (4.15), we have

$$
\begin{aligned}
\left\|x_{n+1}-p\right\| & =\left\|\alpha_{n}\left(f\left(x_{n}\right)-p\right)+\left(1-\alpha_{n}\right)\left(z_{n}-p\right)\right\| \leq\left(1-\alpha_{n}\right)\left\|z_{n}-p\right\|+\alpha_{n}\left\|f\left(x_{n}\right)-p\right\| \\
& \leq\left(1-\alpha_{n}\right)\left\|x_{n}-p\right\|+\alpha_{n} \alpha\left\|x_{n}-p\right\|+\alpha_{n}\|f(p)-p\| \\
& =\left(1-\alpha_{n}(1-\alpha)\right)\left\|x_{n}-p\right\|+\alpha_{n}(1-\alpha) \frac{\|f(p)-p\|}{1-\alpha},
\end{aligned}
$$

which implies that

$$
\left\|x_{n}-p\right\| \leq \max \left\{\left\|x_{1}-p\right\|, \frac{\|f(p)-p\|}{1-\alpha}\right\}, \quad \forall n \in \mathbb{N},
$$

so $\left\{x_{n}\right\}$ is bounded. Further, $\left\{u_{n}\right\},\left\{w_{n}\right\}$ and $\left\{y_{n}\right\}$ are also bounded by (4.11).

By Lemma 2.5, from (4.10) we have

$$
\begin{aligned}
\left\|u_{n+1}-u_{n}\right\|^{2} & =\left\|T_{r_{n+1}}^{f} x_{n+1}-T_{r_{n}}^{f} x_{n}\right\|^{2} \\
& \leq\left(\left\|x_{n+1}-x_{n}\right\|+\frac{\left|r_{n}-r_{n+1}\right|}{r_{n}}\left\|T_{r_{n}}^{f} x_{n}-x_{n}\right\|\right)^{2} \\
& \leq\left\|x_{n+1}-x_{n}\right\|^{2}+\frac{\left|r_{n}-r_{n+1}\right|}{r_{n}} M_{1}, \\
\left\|w_{n+1}-w_{n}\right\|^{2} & =\left\|T_{r_{n+1}}^{g} A u_{n+1}-T_{r_{n}}^{g} A u_{n}\right\|^{2} \\
& \leq\left(\left\|A u_{n+1}-A u_{n}\right\|+\frac{\left|r_{n}-r_{n+1}\right|}{r_{n}}\left\|T_{r_{n}}^{g} A u_{n}-A u_{n}\right\|\right)^{2} \\
& \leq\left\|A u_{n+1}-A u_{n}\right\|^{2}+\frac{\left|r_{n}-r_{n+1}\right|}{r_{n}} M_{1}
\end{aligned}
$$

and

$$
\begin{aligned}
\left\|y_{n+1}-y_{n}\right\|^{2} \leq & \left\|u_{n+1}+\xi B\left(S w_{n+1}-A u_{n+1}\right)-u_{n}-\xi B\left(S w_{n}-A u_{n}\right)\right\|^{2} \\
= & \left\|u_{n+1}-u_{n}+\xi B\left(S w_{n+1}-A u_{n+1}-\left(S w_{n}-A u_{n}\right)\right)\right\|^{2} \\
= & \left\|u_{n+1}-u_{n}\right\|^{2}+\left\|\xi B\left(S w_{n+1}-A u_{n+1}-\left(S w_{n}-A u_{n}\right)\right)\right\|^{2} \\
& +2 \xi\left\langle u_{n+1}-u_{n}, B\left(S w_{n+1}-A u_{n+1}-\left(S w_{n}-A u_{n}\right)\right)\right\rangle \\
\leq & \left\|u_{n+1}-u_{n}\right\|^{2}+\xi^{2}\|B\|^{2}\left\|S w_{n+1}-A u_{n+1}-\left(S w_{n}-A u_{n}\right)\right\|^{2} \\
& +2 \xi\left\langle A\left(u_{n+1}-u_{n}\right), S w_{n+1}-A u_{n+1}-\left(S w_{n}-A u_{n}\right)\right\rangle \\
= & \left\|u_{n+1}-u_{n}\right\|^{2}+\xi^{2}\|B\|^{2}\left\|S w_{n+1}-A u_{n+1}-\left(S w_{n}-A u_{n}\right)\right\|^{2} \\
& +2 \xi\left\langle A\left(u_{n+1}-u_{n}\right)+S w_{n+1}-A u_{n+1}-\left(S w_{n}-A u_{n}\right), S w_{n+1}\right. \\
& \left.-A u_{n+1}-\left(S w_{n}-A u_{n}\right)\right\rangle \\
& -2 \xi\left\langle S w_{n+1}-A u_{n+1}-\left(S w_{n}-A u_{n}\right), S w_{n+1}-A u_{n+1}-\left(S w_{n}-A u_{n}\right)\right\rangle \\
= & \left\|u_{n+1}-u_{n}\right\|^{2}+\xi^{2}\|B\|^{2}\left\|S w_{n+1}-A u_{n+1}-\left(S w_{n}-A u_{n}\right)\right\|^{2} \\
& +2 \xi\left\langle S w_{n+1}-S w_{n}, S w_{n+1}-A u_{n+1}-\left(S w_{n}-A u_{n}\right)\right\rangle \\
& -2 \xi\left\|S w_{n+1}-A u_{n+1}-\left(S w_{n}-A u_{n}\right)\right\|^{2}
\end{aligned}
$$




$$
\begin{aligned}
& =\left\|u_{n+1}-u_{n}\right\|^{2}+\xi^{2}\|B\|^{2}\left\|S w_{n+1}-A u_{n+1}-\left(S w_{n}-A u_{n}\right)\right\|^{2} \\
& +2 \xi \frac{1}{2}\left\{\left\|S w_{n+1}-S w_{n}\right\|^{2}+\left\|S w_{n+1}-A u_{n+1}-\left(S w_{n}-A u_{n}\right)\right\|^{2}\right. \\
& \left.-\left\|A u_{n+1}-A u_{n}\right\|^{2}\right\} \\
& -2 \xi\left\|S w_{n+1}-A u_{n+1}-\left(S w_{n}-A u_{n}\right)\right\|^{2} \\
& =\left\|u_{n+1}-u_{n}\right\|^{2}+\xi^{2}\|B\|^{2}\left\|S w_{n+1}-A u_{n+1}-\left(S w_{n}-A u_{n}\right)\right\|^{2} \\
& +\xi\left\{\left\|S w_{n+1}-S w_{n}\right\|^{2}-\left\|A u_{n+1}-A u_{n}\right\|^{2}\right\} \\
& -\xi\left\|S w_{n+1}-A u_{n+1}-\left(S w_{n}-A u_{n}\right)\right\|^{2} \\
& \leq\left\|u_{n+1}-u_{n}\right\|^{2}-\xi\left(1-\xi\|B\|^{2}\right)\left\|S w_{n+1}-A u_{n+1}-\left(S w_{n}-A u_{n}\right)\right\|^{2} \\
& +\xi\left\{\left\|w_{n+1}-w_{n}\right\|^{2}-\left\|A u_{n+1}-A u_{n}\right\|^{2}\right\} \\
& \leq\left\|u_{n+1}-u_{n}\right\|^{2}-\xi\left(1-\xi\|B\|^{2}\right)\left\|S w_{n+1}-A u_{n+1}-\left(S w_{n}-A u_{n}\right)\right\|^{2} \\
& +\xi\left\{\left\|A u_{n+1}-A u_{n}\right\|^{2}+\frac{\left|r_{n}-r_{n+1}\right|}{r_{n}} M_{1}-\left\|A u_{n+1}-A u_{n}\right\|^{2}\right\} \\
& =\left\|u_{n+1}-u_{n}\right\|^{2}-\xi\left(1-\xi\|B\|^{2}\right)\left\|S w_{n+1}-A u_{n+1}-\left(S w_{n}-A u_{n}\right)\right\|^{2} \\
& +\xi \frac{\left|r_{n}-r_{n+1}\right|}{r_{n}} M_{1} \\
& \leq\left\|x_{n+1}-x_{n}\right\|^{2}-\xi\left(1-\xi\|B\|^{2}\right)\left\|S w_{n+1}-A u_{n+1}-\left(S w_{n}-A u_{n}\right)\right\|^{2} \\
& +\frac{\left|r_{n}-r_{n+1}\right|}{r_{n}}\left(\xi M_{1}+M_{1}\right)
\end{aligned}
$$

where $M_{1}$ is a constant satisfying

$$
\begin{aligned}
& \sup _{n \in \mathbb{N}}\left\{2\left\|x_{n+1}-x_{n}\right\|\left\|T_{r_{n}}^{f} x_{n}-x_{n}\right\|+\frac{\left|r_{n}-r_{n+1}\right|}{r_{n}}\left\|T_{r_{n}}^{f} x_{n}-x_{n}\right\|^{2},\right. \\
& \left.2\left\|A u_{n+1}-A u_{n}\right\|\left\|T_{r_{n}}^{g} A u_{n}-A u_{n}\right\|+\frac{\left|r_{n}-r_{n+1}\right|}{r_{n}}\left\|T_{r_{n}}^{g} A u_{n}-A u_{n}\right\|^{2}\right\} \leq M_{1} .
\end{aligned}
$$

Proving $\left\|x_{n+1}-x_{n}\right\| \rightarrow 0$ as $n \rightarrow \infty$. Setting $\beta_{n}=1-\left(1-\alpha_{n}\right)(1-r)$ and $v_{n}=\frac{x_{n+1}-x_{n}+\beta_{n} x_{n}}{\beta_{n}}$, namely $v_{n}=\frac{\alpha_{n} f\left(x_{n}\right)+\left(1-\alpha_{n}\right) r T y_{n}}{\beta_{n}}$. Let $M_{2}$ be a constant satisfying $\sup _{n \in \mathbb{N}}\left\{\left\|\frac{f\left(x_{n+1}\right)}{\beta_{n+1}}\right\|,\left\|\frac{f\left(x_{n}\right)}{\beta_{n}}\right\|\right.$, $\left.\left\|T y_{n}\right\|\right\} \leq M_{2}$ for all $n \in \mathbb{N}$. Then

$$
\begin{aligned}
\left\|v_{n+1}-v_{n}\right\|= & \left\|\frac{\alpha_{n+1} f\left(x_{n+1}\right)+\left(1-\alpha_{n+1}\right) r T y_{n+1}}{\beta_{n+1}}-\frac{\alpha_{n} f\left(x_{n}\right)+\left(1-\alpha_{n}\right) r T y_{n}}{\beta_{n}}\right\| \\
\leq & \alpha_{n+1}\left\|\frac{f\left(x_{n+1}\right)}{\beta_{n+1}}\right\|+\alpha_{n}\left\|\frac{f\left(x_{n}\right)}{\beta_{n}}\right\|+r\left\|\frac{\left(1-\alpha_{n+1}\right) T y_{n+1}}{\beta_{n+1}}-\frac{\left(1-\alpha_{n}\right) T y_{n}}{\beta_{n}}\right\| \\
\leq & \left(\alpha_{n+1}+\alpha_{n}\right) M_{2}+r \| \frac{\left(1-\alpha_{n+1}\right)\left(T y_{n+1}-T y_{n}\right)}{\beta_{n+1}} \\
& +\frac{\left(1-\alpha_{n+1}\right) T y_{n}}{\beta_{n+1}}-\frac{\left(1-\alpha_{n}\right) T y_{n}}{\beta_{n}} \| \\
\leq & \left(\alpha_{n+1}+\alpha_{n}\right) M_{2}+r \frac{\left(1-\alpha_{n+1}\right)\left\|y_{n+1}-y_{n}\right\|}{\beta_{n+1}}+\left|\frac{\left(1-\alpha_{n+1}\right)}{\beta_{n+1}}-\frac{\left(1-\alpha_{n}\right)}{\beta_{n}}\right| M_{2}
\end{aligned}
$$




$$
\begin{aligned}
= & \left(\alpha_{n+1}+\alpha_{n}\right) M_{2}+r \frac{\left(1-\alpha_{n+1}\right)\left\|y_{n+1}-y_{n}\right\|}{\beta_{n+1}} \\
& +\left|\frac{(1-r)\left(\alpha_{n}-\alpha_{n+1}\right)+\beta_{n+1} \alpha_{n}-\beta_{n} \alpha_{n+1}}{\beta_{n} \beta_{n+1}}\right| M_{2} \\
\leq & \left(\alpha_{n+1}+\alpha_{n}\right) M_{2}+r \frac{\left(1-\alpha_{n+1}\right)\left\|y_{n+1}-y_{n}\right\|}{\beta_{n+1}}+2 \frac{\alpha_{n}+\alpha_{n+1}}{\beta_{n} \beta_{n+1}} M_{2} \\
:= & \rho_{n}+r \frac{\left(1-\alpha_{n+1}\right)\left\|y_{n+1}-y_{n}\right\|}{\beta_{n+1}} .
\end{aligned}
$$

From (4.18) and (4.19), we have

$$
\begin{aligned}
\left\|v_{n+1}-v_{n}\right\|^{2} \leq & \left(\rho_{n}+r \frac{\left(1-\alpha_{n+1}\right)\left\|y_{n+1}-y_{n}\right\|}{\beta_{n+1}}\right)^{2} \\
= & \rho_{n}^{2}+2 \rho_{n} r \frac{\left(1-\alpha_{n+1}\right)\left\|y_{n+1}-y_{n}\right\|}{\beta_{n+1}}+r^{2} \frac{\left(1-\alpha_{n+1}\right)^{2}\left\|y_{n+1}-y_{n}\right\|^{2}}{\beta_{n+1}^{2}} \\
\leq & \rho_{n}^{2}+2 \rho_{n} r \frac{\left(1-\alpha_{n+1}\right)\left\|y_{n+1}-y_{n}\right\|}{\beta_{n+1}}+r^{2} \frac{\left(1-\alpha_{n+1}\right)^{2}}{\beta_{n+1}^{2}}\left\|x_{n+1}-x_{n}\right\|^{2} \\
& +r^{2} \frac{\left(1-\alpha_{n+1}\right)^{2}}{\beta_{n+1}^{2}} \frac{\left|r_{n}-r_{n+1}\right|}{r_{n}}(1+\xi) M_{1} .
\end{aligned}
$$

By the conditions (1) and (2) and (4.20), we obtain

$$
\limsup _{n \rightarrow \infty}\left\{\left\|v_{n+1}-v_{n}\right\|^{2}-\left\|x_{n+1}-x_{n}\right\|^{2}\right\} \leq 0
$$

Notice $\left\|v_{n+1}-v_{n}\right\|^{2}-\left\|x_{n+1}-x_{n}\right\|^{2}=\left(\left\|v_{n+1}-v_{n}\right\|-\left\|x_{n+1}-x_{n}\right\|\right)\left(\left\|v_{n+1}-v_{n}\right\|+\left\|x_{n+1}-x_{n}\right\|\right)$, hence from (4.21) we have

$$
\limsup _{n \rightarrow \infty}\left\{\left\|v_{n+1}-v_{n}\right\|-\left\|x_{n+1}-x_{n}\right\|\right\} \leq 0
$$

By Lemma 2.7 and (4.22), we have $\lim _{n \rightarrow \infty}\left\|v_{n}-x_{n}\right\|=0$, which implies that

$$
\lim _{n \rightarrow \infty}\left\|x_{n+1}-x_{n}\right\|=0
$$

by the definition of $v_{n}$. Since $\left\|x_{n+1}-z_{n}\right\| \rightarrow 0$, together with (4.23), we have

$$
\lim _{n \rightarrow \infty}\left\|x_{n}-z_{n}\right\|=0
$$

Using (4.10), (4.12) and (4.15),

$$
\begin{aligned}
\left\|x_{n+1}-p\right\|^{2} & =\left\|\alpha_{n}\left(f\left(x_{n}\right)-p\right)+\left(1-\alpha_{n}\right)\left(z_{n}-p\right)\right\|^{2} \\
& \leq\left(1-\alpha_{n}\right)\left\|z_{n}-p\right\|^{2}+\alpha_{n}\left\|f\left(x_{n}\right)-p\right\|^{2} \\
& \leq(1-r)\left\|x_{n}-p\right\|^{2}+r\left\|u_{n}-p\right\|^{2}+\alpha_{n}\left\|f\left(x_{n}\right)-p\right\|^{2} \\
& \leq\left\|x_{n}-p\right\|^{2}-r\left\|u_{n}-x_{n}\right\|^{2}+\alpha_{n}\left\|f\left(x_{n}\right)-p\right\|^{2}
\end{aligned}
$$


which yields

$$
\begin{aligned}
r\left\|u_{n}-x_{n}\right\|^{2} & \leq\left\|x_{n}-p\right\|^{2}-\left\|x_{n+1}-p\right\|^{2}+\alpha_{n}\left\|f\left(x_{n}\right)-p\right\|^{2} \\
& =\left(\left\|x_{n}-p\right\|+\left\|x_{n+1}-p\right\|\right)\left(\left\|x_{n}-p\right\|-\left\|x_{n+1}-p\right\|\right)+\alpha_{n}\left\|f\left(x_{n}\right)-p\right\|^{2} \\
& \leq\left(\left\|x_{n}-p\right\|+\left\|x_{n+1}-p\right\|\right)\left\|x_{n}-x_{n+1}\right\|+\alpha_{n}\left\|f\left(x_{n}\right)-p\right\|^{2} .
\end{aligned}
$$

From (4.26) we have

$$
\lim _{n \rightarrow \infty}\left\|T_{r_{n}}^{f} x_{n}-x_{n}\right\|=\lim _{n \rightarrow \infty}\left\|u_{n}-x_{n}\right\|=0
$$

Again, applying (4.25), (4.15) and (4.14), we have

$$
\begin{aligned}
\left\|x_{n+1}-p\right\|^{2} \leq & \left(1-\alpha_{n}\right)\left\|z_{n}-p\right\|^{2}+\alpha_{n}\left\|f\left(x_{n}\right)-p\right\|^{2} \\
\leq & (1-r)\left\|x_{n}-p\right\|^{2}+r\left\|y_{n}-p\right\|^{2}+\alpha_{n}\left\|f\left(x_{n}\right)-p\right\|^{2} \\
\leq & \left\|x_{n}-p\right\|^{2}-r \xi\left(1-\xi\|B\|^{2}\right)\left\|S w_{n}-A u_{n}\right\|^{2} \\
& -r \xi\left\|w_{n}-A u_{n}\right\|^{2}+\alpha_{n}\left\|f\left(x_{n}\right)-p\right\|^{2}
\end{aligned}
$$

which implies that

$$
\begin{aligned}
& r \xi\left(1-\xi\|B\|^{2}\right)\left\|S w_{n}-A u_{n}\right\|^{2}+r \xi\left\|w_{n}-A u_{n}\right\|^{2} \\
& \quad \leq\left\{\left\|x_{n}-p\right\|+\left\|x_{n+1}-p\right\|\right\}\left\|x_{n}-x_{n+1}\right\|+\alpha_{n}\left\|f\left(x_{n}\right)-p\right\|^{2} .
\end{aligned}
$$

From (4.29) we have

$$
\lim _{n \rightarrow \infty}\left\|T_{r_{n}}^{g} A u_{n}-A u_{n}\right\|=\lim _{n \rightarrow \infty}\left\|w_{n}-A u_{n}\right\|=0, \quad \lim _{n \rightarrow \infty}\left\|S w_{n}-A u_{n}\right\|=0
$$

and

$$
\lim _{n \rightarrow \infty}\left\|S w_{n}-w_{n}\right\|=0
$$

Notice $y_{n}=P_{C}\left(u_{n}+\xi B\left(S w_{n}-A u_{n}\right)\right)$ and $u_{n} \in C$ for all $n \in \mathbb{N}$, so

$$
\begin{aligned}
\left\|y_{n}-u_{n}\right\| & =\left\|P_{C}\left(u_{n}+\xi B\left(S w_{n}-A u_{n}\right)\right)-P_{C} u_{n}\right\| \leq\left\|\xi B\left(S w_{n}-A u_{n}\right)\right\| \\
& \leq \xi\|B\|\left\|S w_{n}-A u_{n}\right\|,
\end{aligned}
$$

SO

$$
\lim _{n \rightarrow \infty}\left\|y_{n}-u_{n}\right\|=0
$$

Further, from (4.27), (4.32) and (4.24), we have

$$
\lim _{n \rightarrow \infty}\left\|y_{n}-x_{n}\right\|=0, \quad \lim _{n \rightarrow \infty}\left\|y_{n}-z_{n}\right\|=0
$$


and

$$
\lim _{n \rightarrow \infty}\left\|y_{n}-T y_{n}\right\|=0 \quad \text { by (4.10), (4.24) and (4.33). }
$$

Let $q=P_{\Omega} f(q)$. Choose a subsequence $\left\{x_{n_{k}}\right\}$ such that

$$
\limsup _{n \rightarrow \infty}\left\langle f(q)-q, x_{n}-q\right\rangle=\lim _{k \rightarrow \infty}\left\langle f(q)-q, x_{n_{k}}-q\right\rangle .
$$

Since $\left\{x_{n}\right\}$ is bounded, $\left\{\left\langle f(q)-q, x_{n}-q\right\rangle\right\}$ is bounded. Hence $\limsup _{n \rightarrow \infty}\left\langle f(q)-q, x_{n}-q\right\rangle$ is a constant, namely $\lim _{n \rightarrow \infty}\left\langle f(q)-q, x_{n_{k}}-q\right\rangle$ exists, which implies (4.35) is well defined. Because $\left\{x_{n}\right\}$ is bounded, $\left\{x_{n_{k}}\right\}$ has a weak convergence subsequence which is still denoted by $\left\{x_{n_{k}}\right\}$. Suppose $x_{n_{k}} \rightarrow x^{*}$, we say $x^{*} \in \Omega$. When $x_{n_{k}} \rightarrow x^{*}$, from (4.30), (4.32) and (4.33), we have

$$
u_{n_{k}} \rightarrow x^{*}, \quad y_{n_{k}} \rightarrow x^{*}, \quad z_{n_{k}} \rightarrow x^{*}, \quad A u_{n_{k}} \rightarrow A x^{*}, \quad w_{n_{k}} \rightarrow A x^{*} .
$$

If $T x^{*} \neq x^{*}$, then by (4.34) and (4.36) and Opial's condition, we have

$$
\begin{aligned}
\liminf _{k \rightarrow \infty}\left\|y_{n_{k}}-x^{*}\right\| & <\liminf _{k \rightarrow \infty}\left\|y_{n_{k}}-T x^{*}\right\| \\
& \leq \liminf _{k \rightarrow \infty}\left\{\left\|y_{n_{k}}-T y_{n_{k}}\right\|+\left\|T y_{n_{k}}-T x^{*}\right\|\right\} \\
& \leq \liminf _{k \rightarrow \infty}\left\{\left\|y_{n_{k}}-T y_{n_{k}}\right\|+\left\|y_{n_{k}}-x^{*}\right\|\right\}=\liminf _{k \rightarrow \infty}\left\|y_{n_{k}}-x^{*}\right\|,
\end{aligned}
$$

which is a contradiction, so $T x^{*}=x^{*}$ and $x^{*} \in \mathcal{F}(T)$. Since for each $r>0, E P(f)=\mathcal{F}\left(T_{r}^{f}\right)$ by Lemma 2.2, we have $x^{*} \in \mathcal{F}\left(T_{r}^{f}\right)$. Otherwise, if there exists $r>0$ such that $T_{r}^{f} x^{*} \neq x^{*}$, then by (4.27) and Lemma 2.5 and Opial's condition, we have

$$
\begin{aligned}
\liminf _{k \rightarrow \infty}\left\|x_{n_{k}}-x^{*}\right\| & <\liminf _{k \rightarrow \infty}\left\|x_{n_{k}}-T_{r}^{f} x^{*}\right\| \\
& \leq \liminf _{k \rightarrow \infty}\left\{\left\|x_{n_{k}}-T_{n_{k}}^{f} x_{n_{k}}\right\|+\left\|T_{n_{k}}^{f} x_{n_{k}}-T_{r}^{f} x^{*}\right\|\right\} \\
& =\liminf _{k \rightarrow \infty}\left\|T_{n_{k}}^{f} x_{n_{k}}-T_{r}^{f} x^{* *}\right\| \\
& \leq \liminf _{k \rightarrow \infty}\left\{\left\|x_{n_{k}}-x^{*}\right\|+\frac{\left|r_{n_{k}}-r\right|}{r_{n_{k}}}\left\|T_{n_{k}}^{f} x_{n_{k}}-x_{n_{k}}\right\|\right\} \\
& =\liminf _{k \rightarrow \infty}\left\|x_{n_{k}}-x^{*}\right\|,
\end{aligned}
$$

which is also a contradiction, so $T_{r}^{f} x^{*}=x^{*}$ and $x^{*} \in \mathcal{F}\left(T_{r}^{f}\right)=E P(f)$. Up to now, we have proved $x^{*} \in \mathcal{F}(T) \cap E P(f)$. Similarly, we can also prove $A x^{*} \in \mathcal{F}(S) \cap E P(g)$. Hence $x^{*} \in \Omega$, because of this, we can also obtain

$$
\begin{aligned}
\limsup _{n \rightarrow \infty}\left\langle f(q)-q, x_{n}-q\right\rangle & =\lim _{k \rightarrow \infty}\left\langle f(q)-q, x_{n_{k}}-q\right\rangle \\
& =\left\langle f(q)-q, x^{*}-q\right\rangle \leq 0, \quad \text { where } q=P_{C} f(q) .
\end{aligned}
$$


Finally, we prove the conclusion of this theorem is right. For $q=P_{\Omega} f(q)$, from (4.10) we have

$$
\begin{aligned}
\left\|x_{n+1}-q\right\|^{2}= & \left\|\alpha_{n}\left(h\left(x_{n}\right)-q\right)+\left(1-\alpha_{n}\right)\left(z_{n}-q\right)\right\|^{2} \\
\leq & \left(1-\alpha_{n}\right)^{2}\left\|z_{n}-q\right\|^{2}+2 \alpha_{n}\left\langle h\left(x_{n}\right)-q, x_{n+1}-q\right\rangle \\
\leq & \left(1-\alpha_{n}\right)^{2}\left\|x_{n}-q\right\|^{2}+2 \alpha_{n}\left\langle h\left(x_{n}\right)-h(q)+h(q)-q, x_{n+1}-q\right\rangle \\
\leq & \left(1-\alpha_{n}\right)^{2}\left\|x_{n}-q\right\|^{2}+2 \alpha_{n} \alpha\left\|x_{n}-q\right\|\left\|x_{n+1}-q\right\|+2 \alpha_{n}\left\langle h(q)-q, x_{n+1}-q\right\rangle \\
\leq & \left(1-\alpha_{n}\right)^{2}\left\|x_{n}-q\right\|^{2}+\alpha_{n} \alpha\left\|x_{n}-q\right\|^{2}+\alpha_{n} \alpha\left\|x_{n+1}-q\right\|^{2} \\
& +2 \alpha_{n}\left(h(q)-q, x_{n+1}-q\right\rangle \\
= & \left(1-2 \alpha_{n}\right)\left\|x_{n}-q\right\|^{2}+\alpha_{n}^{2}\left\|x_{n}-q\right\|^{2}+\alpha_{n} \alpha\left\|x_{n}-q\right\|^{2}+\alpha_{n} \alpha\left\|x_{n+1}-q\right\|^{2} \\
& +2 \alpha_{n}\left\langle h(q)-q, x_{n+1}-q\right\rangle .
\end{aligned}
$$

From (4.40) we have

$$
\begin{aligned}
\left\|x_{n+1}-q\right\|^{2} \leq & \left(1-\alpha_{n} \frac{2-2 \alpha}{1-\alpha_{n} \alpha}\right)\left\|x_{n}-q\right\|^{2}+\frac{\alpha_{n}^{2}}{1-\alpha_{n} \alpha}\left\|x_{n}-q\right\|^{2} \\
& +2 \frac{\alpha_{n}}{1-\alpha_{n} \alpha}\left\langle h(q)-q, x_{n+1}-q\right\rangle,
\end{aligned}
$$

by (4.41) and Lemma 2.6, we have $x_{n} \rightarrow q \in \Omega$. Again, from (4.27) and (4.30), we have $u_{n} \rightarrow q \in \Omega$ and $w_{n} \rightarrow A q \in F(S) \cap E P(f)$, respectively. The proof is completed.

\section{Remark}

(1) In this paper, the iterative coefficient $\alpha$ or $r$ can be replaced with the sequence $\left\{\zeta_{n}\right\}$ if $\left\{\zeta_{n}\right\}$ satisfies $\left\{\zeta_{n}\right\} \subset[\varrho, \vartheta]$, where $\varrho, \vartheta \in(0,1)$;

(2) Obviously, if $H_{1}=H_{2}$ in this paper, these weak and strong convergence theorems are also true;

(3) In this paper, if $T$ is a nonexpansive mapping from $H_{1}$ into $H_{1}$ and $f(x, y)$ is a bi-function from $H_{1} \times H_{1}$ into $\mathbb{R}$ with the conditions (A1)-(A4), $S$ is a nonexpansive mapping from $H_{2}$ into $H_{2}$ and $g(u, v)$ is a bi-function from $H_{2} \times H_{2}$ into $\mathbb{R}$ with the conditions (A1)-(A4), then we may obtain a series of similar algorithms.

\section{Competing interests}

The authors declare that they have no competing interests.

Authors' contributions

Both authors contributed equally and significantly in writing this paper. Both authors read and approved the final manuscript.

\section{Author details}

${ }^{1}$ Department of Mathematics, Honghe University, Yunnan, 661100, China. ${ }^{2}$ Department of Mathematics, National Kaohsiung Normal University, Kaohsiung, 824, Taiwan.

\section{Acknowledgements}

The first author was supported by the Natural Science Foundation of Yunnan Province (2010ZC152). The second author was supported partially by grant No. NSC 101-2115-M-017-001 of the National Science Council of the Republic of China. 


\section{References}

1. Blum, E, Oettli, W: From optimization and variational inequalities to equilibrium problems. Math. Stud. 63, 123-145 (1994)

2. Moudafi, A, Théra, M: Proximal and dynamical approaches to equilibrium problems. In: III-Posed Variational Problems and Regularization Techniques (Trier, 1998). Lecture Notes in Economics and Mathematical Systems, vol. 477, pp. 187-201. Springer, Berlin (1999)

3. Flam, SD, Antipin, AS: Equilibrium programming using proximal-link algorithms. Math. Program. 78, $29-41$ (1997)

4. Tada, A, Takahashi, W: Weak and strong convergence theorems for a nonexpansive mapping and an equilibrium problem. J. Optim. Theory Appl. 133, 359-370 (2007)

5. Takahashi, S, Takahashi, W: Viscosity approximation methods for equilibrium problems and fixed point problems in Hilbert spaces. J. Math. Anal. Appl. 331, 506-515 (2007)

6. Chang, SS, Joseph Lee, HW, Chan, CK: A new method for solving equilibrium problem fixed point problem and variational inequality problem with application to optimization. Nonlinear Anal. 70, 3307-3319 (2009)

7. Jung, JS: Strong convergence of composite iterative methods for equilibrium problems and fixed point problems. Appl. Math. Comput. 213, 498-505 (2009)

8. He, Z, Du, W-S: Strong convergence theorems for equilibrium problems and fixed point problems: a new iterative method, some comments and applications. Fixed Point Theory Appl. 2011, Article ID 33 (2011)

9. He, Z: A new iterative scheme for equilibrium problems and fixed point problems of strict pseudo-contractive mappings and its application. Math. Commun. 17, 411-422 (2012)

10. He, Z: The split equilibrium problems and its convergence algorithms. J. Inequal. Appl. 2012, Article ID 162 (2012)

11. He, Z, Du, W-S: Nonlinear algorithms approach to split common solution problems. Fixed Point Theory Appl. 2012, Article ID 130 (2012)

12. Combettes, PL, Hirstoaga, A: Equilibrium programming in Hilbert spaces. J. Nonlinear Convex Anal. 6, 117-136 (2005)

13. Xu, HK: An iterative approach to quadratic optimization. J. Optim. Theory Appl. 116, 659-678 (2003)

14. Suzuki, T: Strong convergence theorems for infinite families of nonexpansive mappings in general Banach spaces. Fixed Point Theory Appl. 2005(1), 103-123 (2005)

15. Moudafi, A: Viscosity approximation methods for fixed-points problems. J. Math. Anal. Appl. 241, 46-55 (2000)

16. Xu, H-K: Viscosity approximation methods for nonexpansive mappings. J. Math. Anal. Appl. 298, 279-291 (2004)

doi:10.1186/1687-1812-2013-47

Cite this article as: He and Du: On hybrid split problem and its nonlinear algorithms. Fixed Point Theory and Applications 2013 2013:47.

\section{Submit your manuscript to a SpringerOpen ${ }^{\ominus}$ journal and benefit from:}

- Convenient online submission

- Rigorous peer review

- Immediate publication on acceptance

- Open access: articles freely available online

- High visibility within the field

- Retaining the copyright to your article 